\title{
Gas Plasma-Conditioned Ringer's Lactate Enhances the Cytotoxic Activity of Cisplatin and Gemcitabine in Pancreatic Cancer In Vitro and In Ovo
}

\author{
Kim-Rouven Liedtke $\left.{ }^{1,+}{ }^{(}\right)$, Eric Freund $\left.{ }^{1,2,+}{ }^{(}\right)$, Maraike Hermes ${ }^{1,2}$, Stefan Oswald ${ }^{3}(\mathbb{D}$, \\ Claus-Dieter Heidecke ${ }^{1}$, Lars-Ivo Partecke ${ }^{1,4}$ and Sander Bekeschus ${ }^{2, *(D)}$ \\ 1 Department of General, Visceral, Thoracic and Vascular Surgery, Greifswald University Medical Center, \\ 17475 Greifswald, Germany; Kim.Liedtke@med.uni-greifswald.de (K.-R.L.); eric.freund@inp-greifswald.de (E.F.); \\ maraike.hermes@stud.uni-greifswald.de (M.H.); Claus-Dieter.Heidecke@med.uni-greifswald.de (C.-D.H.); \\ Ivo.Partecke@med.uni-greifswald.de (L.-I.P.) \\ 2 Centre for Innovation Competence (ZIK) plasmatis, Leibniz Institute for Plasma Science and Technology \\ (INP Greifswald), 17489 Greifswald, Germany \\ 3 Department of Clinical Pharmacology, Greifswald University Medical Center, 17475 Greifswald, Germany; \\ stefan.oswald@uni-greifswald.de \\ 4 General-, Visceral-, and Thoracic Surgery, Helios Clinic Schleswig, 24837 Schleswig, Germany \\ * Correspondence: sander.bekeschus@inp-greifswald.de \\ + Authors contributed equally to the work as first authors.
}

Received: 28 November 2019; Accepted: 27 December 2019; Published: 2 January 2020

\begin{abstract}
Pancreatic cancer is one of the most aggressive tumor entities. Diffuse metastatic infiltration of vessels and the peritoneum restricts curative surgery. Standard chemotherapy protocols include the cytostatic drug gemcitabine with limited efficacy at considerable toxicity. In search of a more effective and less toxic treatment modality, we tested in human pancreatic cancer cells (MiaPaca and PaTuS) a novel combination therapy consisting of cytostatic drugs (gemcitabine or cisplatin) and gas plasma-conditioned Ringer's lactate that acts via reactive oxygen species. A decrease in metabolic activity and viability, change in morphology, and cell cycle arrest was observed in vitro. The combination treatment was found to be additively toxic. The findings were validated utilizing an in ovo tumor model of solid pancreatic tumors growing on the chorion-allantois membrane of fertilized chicken eggs (TUM-CAM). The combination of the drugs (especially cisplatin) with the plasma-conditioned liquid significantly enhanced the anti-cancer effects, resulting in the induction of cell death, cell cycle arrest, and inhibition of cell growth with both of the cell lines tested. In conclusion, our novel combination approach may be a promising new avenue to increase the tolerability and efficacy of locally applied chemotherapeutic in diffuse metastatic peritoneal carcinomatosis of the pancreas.
\end{abstract}

Keywords: anticancer drugs; combination therapy; kINPen; plasma medicine; reactive oxygen and nitrogen species; ROS

\section{Introduction}

With approximately 18.1 million new cases diagnosed and about 9.6 million deaths in 2018, cancer is one of the most significant global medical challenges of our time [1]. It represents the most common cause of death in the western world within the younger population below the age of 80 [2]. Numerous improvements in the diagnosis and treatment of cancer, notwithstanding, pancreatic cancer (PC) remains one of the most lethal cancers with an almost equally incidence and mortality [3]. Due to non-specific symptoms in the early stages of the disease, the diagnosis is often made late in patients 
already suffering from advanced metastatic disease [4]. Even with advanced surgery (e.g., vessel reconstruction and neoadjuvant chemotherapy), only about a quarter of patients are suitable for curative surgery $[5,6]$. With maximal surgery and adjuvant chemotherapy (chemotherapy $=\mathrm{CTx}$ ), the five-year survival rate is nonetheless unsatisfactorily low with $17.5-28.8 \%$ [7,8]. In addition, the incidence of PC is on the rise, and by 2030, it is expected to be the second most common cause of cancer-related deaths [9].

Due to the unsatisfactory clinical results, new therapeutic approaches are urgently needed. The combination of chemotherapeutics with reactive oxygen species (ROS) or ROS-producing drugs has long been debated as an exciting approach in cancer therapy [10]. Among the technical approach to locally generate ROS is photodynamic therapy, mainly generating singlet oxygen [11], and laser treatment of pancreatic cancer. The laser treatment does produce significant anticancer effects; however, it is unpractical in its clinical application [12]. Another option to generate multiple ROS is the application of cold physical plasma. Not to be confused with blood plasma, gas plasmas are electrically neutral gases that can be generated at tissue-compatible temperatures of about $40{ }^{\circ} \mathrm{C}$. As a result of the (partial) ionization, ambient air serves as a reservoir to generate vast amounts of ROS [13].

Several groups, including us, have previously demonstrated anti-tumor effects with physical plasma treatment [14-16]. Some studies already highlighted an added value when combining direct plasma treatment with chemotherapeutic [17-20]. Apart from the possibility of directly treating cells or tissues, significant efforts have also been undertaken to utilize the therapeutic capacity of liquids exposed to gas plasmas [21]. While initially, cell culture medium was used predominantly for this purpose in vitro and even in vivo [22,23], it became increasingly clear that only clinically relevant liquids such as sodium chloride and Ringer's lactate harbor the translational potential to improve therapeutic outcomes in preclinical and clinical models [24]. Ringer's lactate solution or liquid showed to be especially promising candidates because central mediators such as hydrogen peroxide $\left(\mathrm{H}_{2} \mathrm{O}_{2}\right)$ are stable over weeks while the lactate serves as an essential bystander for the anti-cancer effects observed [25].

Most standard cancer therapies involved one or several oncological treatments based on decades of clinical experience, i.e., surgery, radiation, immunotherapy/targeted therapy, and chemotherapy. With ever more combination therapies emerging at the clinical horizon, it seems clear that the value of plasma-conditioned liquids likely is to serve as an additive compound to existing therapies. One example is the hyperthermic intraperitoneal chemotherapy (HIPEC), where a chemotherapeutic agent dissolved into a liquid is perfused in the peritoneal cavity to locally attack metastatic tumor nodes in conjunction with cytoreductive surgery [26]. There is a great need for increasing efficacy and decreasing the side effects of this therapy based on the drugs commonly used [27]. To this end, we investigated the combined effect of plasma-conditioned liquid and the HIPEC drugs cisplatin and gemcitabine in pancreatic cancer cells in vitro (two-dimensional monolayers) and in ovo (three-dimensional tumor with blood supply and matrix remodeling). Promising additive tumor-toxicity was observed that might optimize intraperitoneal perfusion in future therapies of pancreatic cancers or peritoneal carcinomatosis by reducing drug concentrations and thereby decreasing side effects while maintaining a similar efficacy.

\section{Results}

\subsection{Cisplatin, Gemcitabine, and Plasma-Conditioned Ringer's Lactate Inactivated Pancreatic Cancer Cells in a Dose-Dependent Manner}

To test the effect of cisplatin or gemcitabine in combination with physical plasma-conditioned Ringer's lactate (RiLac), the first step was to identify the optimal concentration of each compound to reach a $25 \%$ reduction in the cancer cells metabolic activity (IC-25). The metabolic activity of the cells is analyzed utilizing their capacity to reduce resazurin to the fluorescent resorufin. The transformation of this metabolite correlates with the cells' metabolic activity, so it can be used to describe the percentage of cancer cells that were inactivated through the different treatment regimens [28]. Cisplatin inactivated 
MiaPaca and PaTuS human pancreatic cancer cells in a dose-dependent manner (Figure 1a). The PaTuS cancer cells were more resistant to both drugs, and to reach the IC-25, $50 \mu \mathrm{M}$ of cisplatin were needed, while a similar effect was reached with $25 \mu \mathrm{M}$ of gemcitabine. The toxic effect of gemcitabine was detectable at lower concentrations (Figure 1b). As the next step, an oxidative liquid was generated using cold physical plasma (Figure 1c) using Ringer's lactate (RiLac). RiLac is a well known and clinically applied liquid and could already proof in a previous study to be an excellent candidate to store physical plasma-derived oxidants [24]. The idea was to combine this liquid with chemotherapy in two ways. The oxidative liquid was applied before chemotherapy to investigate sensitization to chemotherapy with oxidative stress (plasma-CTx), or chemotherapy was added first to sensitize cells to oxidative stress (CTx-plasma). One crucial mediator of the effect of plasma-conditioned liquid is hydrogen peroxide $\left(\mathrm{H}_{2} \mathrm{O}_{2}\right)$ that was deposited in RiLac through the exposure to the effluent of the kINPen argon-plasma jet in a treatment time-dependent fashion (Figure 1d). Treatment times of $60 \mathrm{~s}$ generated $200 \mu \mathrm{M}$ of $\mathrm{H}_{2} \mathrm{O}_{2}$ while $120 \mathrm{~s}$ generated approximately $300 \mu \mathrm{M}$. To calculate reliable values of generated hydrogen peroxide, the amount of evaporated liquid during these long plasma treatment times was supplemented with double-distilled water (Supplementary Figure S1a). Besides $\mathrm{H}_{2} \mathrm{O}_{2}$, the time-dependent deposition of nitrate and to a greater extent nitrite was detected in plasma-conditioned RiLac (Figure 1e). The oxidational capacity of the plasma-conditioned RiLac was validated via oxidation of the hydroxyl radical and peroxynitrite anion indicator hydroxyphenyl fluorescein (HPF, Figure 1f). Ringer's lactate is an ideal oxidative solution, it lacks buffer capacity and long plasma-treatment times could induce a drop in the $\mathrm{pH}$ level of the liquid. However, 120 of the exposure of RiLac to gas plasma only modest decreased the $\mathrm{pH}$, which was also the case for a combinational regimen with cisplatin and gemcitabine (Figure $1 \mathrm{~g}$ ). MiaPaca cells reached the 25\% reduction of their metabolic activity after exposure to $60 \mathrm{~s}$ plasma-conditioned RiLac, while PaTuS needed $120 \mathrm{~s}$ (Figure 1e) to reach the target IC-25. These experiments defined the treatment modalities that were kept constant throughout all further experiments in this study (Table 1).

\subsection{Combination of Cisplatin and Gemcitabine with Physical Plasma-Conditioned Ringer's Lactate Enhanced the Inactivation of Pancreatic Cancer Cells}

To next identify the benefit of combination therapy, cisplatin or gemcitabine were combined with physical plasma-conditioned RiLac and compared to the respective single treatment regimens. For this, cells were exposed to one CTx, plasma-RiLac, or RiLac alone for $30 \mathrm{~min}$ before the respective liquid was removed and exchanged with the cell culture medium. After $24 \mathrm{~h}$ of culture, the respective second treatment was performed, and the cells were now exposed to the complementary treatment as foreseen in the combination therapy regimen (Figure 2a). The downstream analysis of the cells was performed $24 \mathrm{~h}$ after the addition of the second treatment or control condition (i.e., $48 \mathrm{~h}$ after exposure to the first treatment or control condition). Plasma-condition RiLac and CTx alone showed a modest but significant reduction of the metabolic activity in MiaPaca (Figure 2b) but not PaTuS (Figure 2c) pancreatic cancer cells. In contrast to these results, the combination of gemcitabine and cisplatin with plasma-RiLac (in both plasma-CTx and CTx-plasma treatment protocols) induced a significant inactivation of PaTuS cells (Figure 2c) with cisplatin having a stronger combination effect compared to gemcitabine. This was also observed in MiaPaca cells that, in general, responded stronger to combination treatment with CTX and plasma-RiLac (Figure 2b) as compared to the other regimens and PaTuS cells, respectively. The concentration of hydrogen peroxide was measured in the combinational regimen, showing only less difference to the mono treatment with plasma-conditioned RiLac, and also in wells that received fresh plasma-RiLac 30 min before (Figure 2d). This showed a 50\% decrease in $\mathrm{H}_{2} \mathrm{O}_{2}$ through the reaction with the cancer cells. In order to gain more knowledge about the vital role of $\mathrm{H}_{2} \mathrm{O}_{2}$ in mediating the cytotoxic effect experiments were carried out were $\mathrm{H}_{2} \mathrm{O}_{2}$ was supplemented to RiLac in the same amount generated through the plasma-treatment. The combinational regimen, containing $\mathrm{H}_{2} \mathrm{O}_{2}$ only, also diminished the cancer cells' metabolic activity but was less effective (Supplementary Figure S1b,c). In further control experiments, the $\mathrm{H}_{2} \mathrm{O}_{2}$-scavenging enzyme catalase was added to all treatment 
regimens and completely diminished the effect of plasma-conditioned RiLac, validating the critical role of $\mathrm{H}_{2} \mathrm{O}_{2}$ for the plasma effect (Supplementary Figure S1d-g). Interestingly, the scavenging could not fully prevent the effect when plasma-conditioned RiLac was applied in combination with CTx.

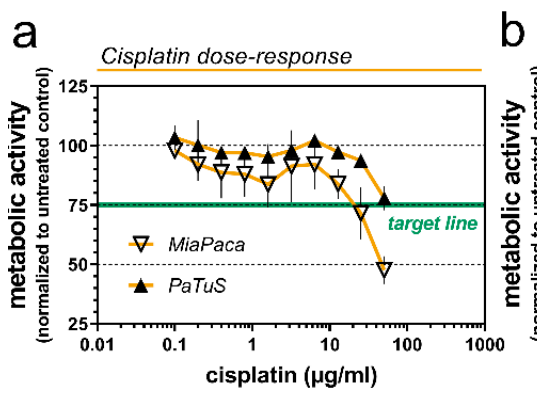

b
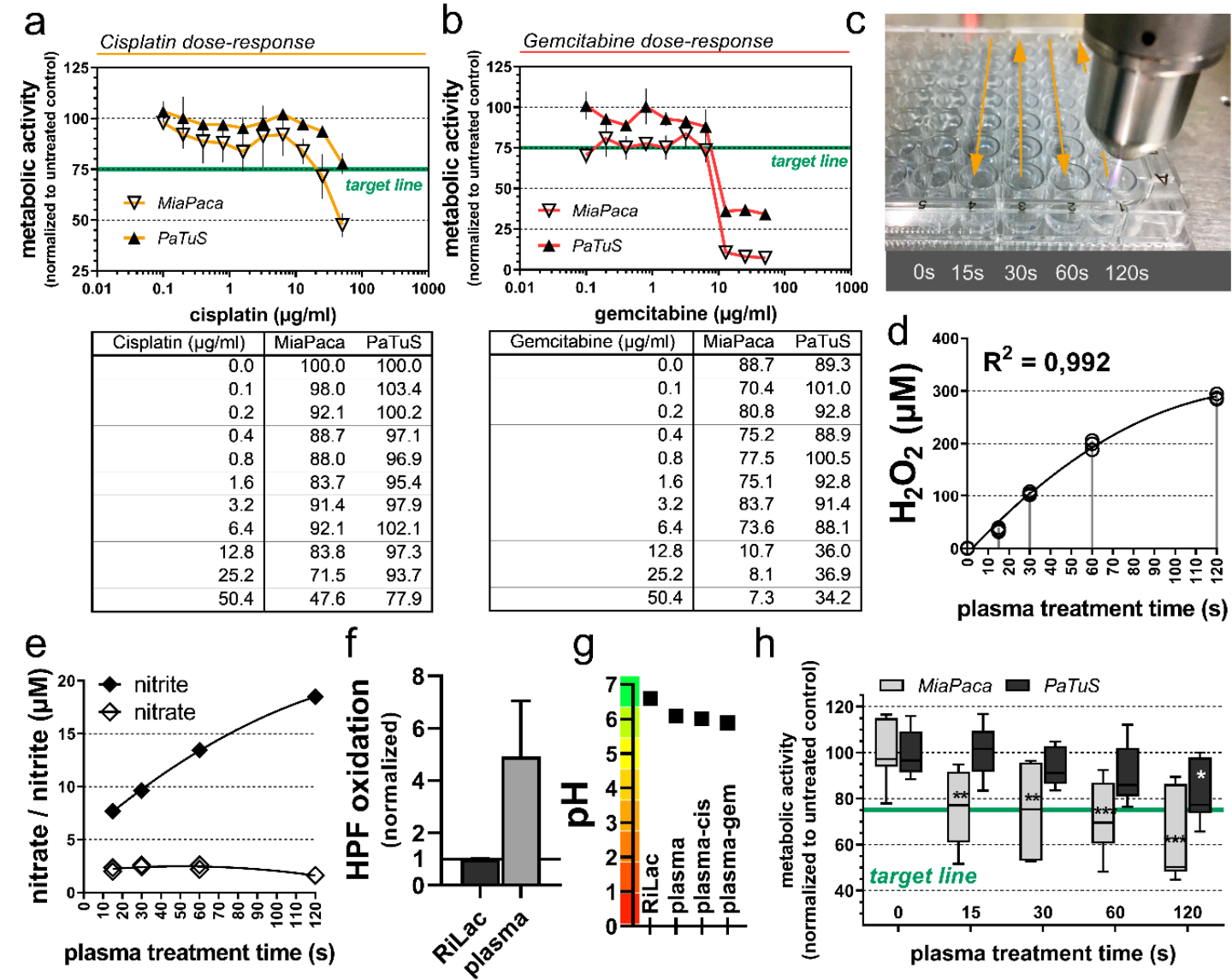

h

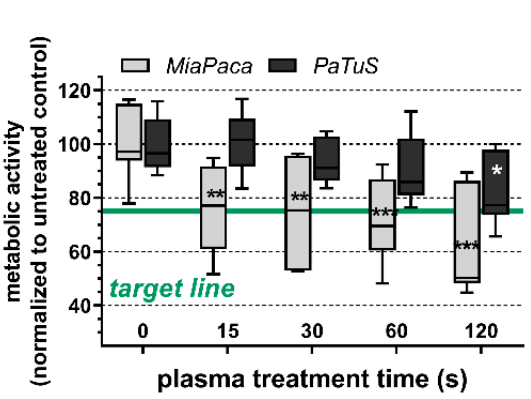

Figure 1. Dose-depended inactivation of pancreatic cancer cells through the application of cisplatin, gemcitabine, or physical plasma-conditioned Ringer's lactate. $(\mathbf{a}, \mathbf{b})$ the metabolic activity of MiaPaca and PaTuS pancreatic cancer cells $24 \mathrm{~h}$ post-exposure to (a) cisplatin and (b) gemcitabine IC-25 target-line (green); (c) schematic overview of the standardized exposure of $100 \mu \mathrm{L}$ Ringer's lactate to the effluent of a kINPen argon plasma jet for 0, 15, 30, 60, and $120 \mathrm{~s}$, and (d) the amount of hydrogen peroxide $\left(\mathrm{H}_{2} \mathrm{O}_{2}\right)$ generated in this liquid; (e) metabolic activity of MiaPaca and PaTuS pancreatic cancer cells $24 \mathrm{~h}$ post-exposure to the respective physical plasma-conditioned Ringer's lactate. Data are $(\mathbf{a}, \mathbf{b})$ mean $\pm \mathrm{SD}$ and are representatives out of three independent experiments, (d) individual technical replicates with curve fitting of a quadratic function $\left(\mathrm{R}^{2}=0.992\right)$ from three technical replicates; (e) concentration of nitrate and nitrite in plasma-conditioned liquid; (f) oxidation of hydroxyphenyl fluorescein (HPF) in Ringer's lactate through $120 \mathrm{~s}$ of plasma-treatment; (g) pH value of $120 \mathrm{~s}$ plasma-conditioned Ringer's lactate and plasma in combination with cisplatin and gemcitabine; (h) metabolic activity of pancreatic cancer cells with IC-25 target-line (green) $24 \mathrm{~h}$ post-exposure to physical plasma-conditioned Ringer's lactate as representative out of three independent experiments showing median and min to max. Statistical significance was calculated utilizing ANOVA.

Table 1. Concentration of adjuvant chemotherapy (CTx) and plasma-conditioned Ringer's lactate used in this study.

\begin{tabular}{cccc}
\hline \multicolumn{4}{c}{ Drug Concentrations or Plasma-Exposure Times to $\mathbf{1 0 0} \mu \mathrm{L}$ of Treatment Liquid for in vitro and in ovo } \\
\multicolumn{4}{c}{ Experiments to Reach at Least IC-25 } \\
\hline Component & Cisplatin & Gemcitabine & Plasma-conditioned Ringer's Lactate \\
\hline MiaPaca & $25 \mu \mathrm{M}$ & $50 \mu \mathrm{M}$ & $60 \mathrm{~s} / 100 \mu \mathrm{L}$ \\
PaTuS & $50 \mu \mathrm{M}$ & $50 \mu \mathrm{M}$ & $120 \mathrm{~s} / 100 \mu \mathrm{L}$ \\
\hline
\end{tabular}




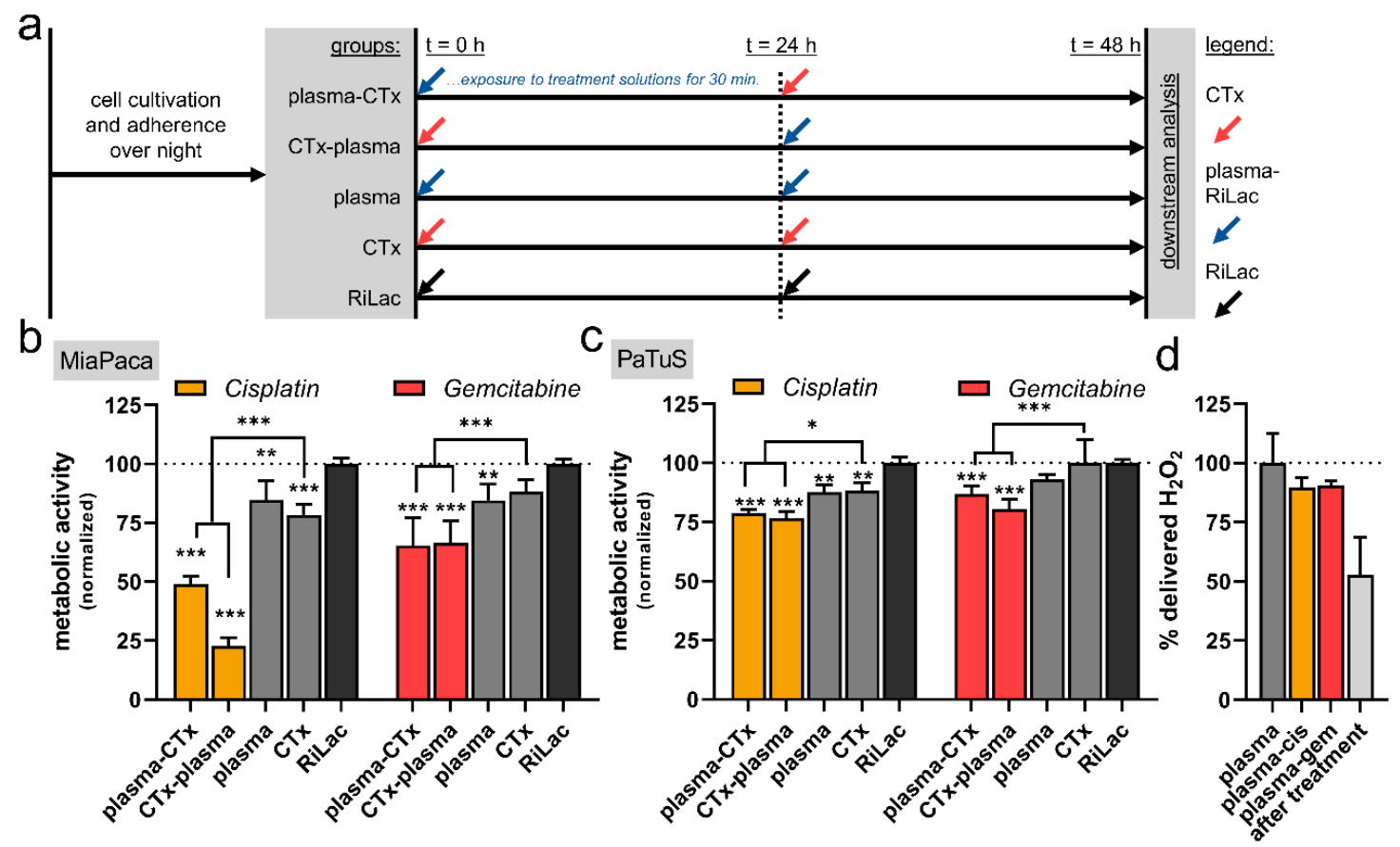

Figure 2. Additive decrease of metabolic activity using cisplatin or gemcitabine with physical plasma-conditioned Ringer's lactate in pancreatic cancer cells. (a) Experimental procedure of the in vitro treatment regimens with the application of different treatment liquids (30 min exposure time; CTx = chemotherapeutic agents, plasma = physical-plasma-conditioned Ringer's lactate, RiLac $=$ Ringer's lactate before the solutions were replaced with fresh cell culture medium) at $0 \mathrm{~h}$ and $24 \mathrm{~h}$, as well as downstream analysis at $48 \mathrm{~h}$; (b,c) the metabolic activity at $48 \mathrm{~h}$ of (b) MiaPaca and (c) PaTuS pancreatic cancer cells; (d) percent of the amount of $\mathrm{H}_{2} \mathrm{O}_{2}$ initially induced through the plasma treatment in the combinational regimen and post-exposure to plasma-conditioned RiLac. Data $(\mathbf{b}, \mathbf{c})$ are representatives out of eight independent experiments, or (d) representatives showing mean +SD. Statistical significance was calculated utilizing ANOVA.

\subsection{Combination of Cisplatin and Gemcitabine with Physical Plasma-Conditioned Ringer's Lactate Mediated Terminal Cell Death to Pancreatic Cancer Cells}

To analyze whether the decrease in metabolic activity was concomitant with terminal cell death, cells were harvested after control, single, or combination treatment at $48 \mathrm{~h}$. Using DAPI as nuclear counterstain allowing the identification of cells with compromised cellular membranes, and a dye identifying the presence of active caspases within cells, it was possible to distinguish between viable $\left(\mathrm{DAPI}^{-}\right.$, caspase $\left.{ }^{-}\right)$, early apoptotic $\left(\mathrm{DAPI}^{-}\right.$, caspase $\left.^{+}\right)$, late apoptotic $\left(\mathrm{DAPI}^{+}\right.$, caspase $\left.^{+}\right)$and necrotic $\left(\mathrm{DAPI}^{+}\right.$, caspase ${ }^{-}$) MiaPaca (Figure 3a) and PaTuS (Figure 3b) cells. All treatment regimen reduced the viability of the MiaPaca (Figure 3c) and PaTuS (Figure 3d) cells. The single-agent treatment regimens only modestly reduced the fraction of viable cells, while the reduction was much greater with combination treatment in both cell lines. The most effective regimen in PaTuS was plasma-cisplatin (viability $=76.3 \%$ ) and cisplatin-plasma $(83.3 \%$ ) followed by gemcitabine-plasma (viability $=76.4 \%$ ) and plasma-gemcitabine (78.8\%) (Figure 3d). In general, responses in MiaPaca cells were greater with plasma-cisplatin (viability $=31.4 \%$ ) or cisplatin-plasma $(37.6 \%$ ) (Figure $3 c$ ). The combination with gemcitabine was weaker compared to that of cisplatin in MiaPaca cells with viability decreasing to $64.7 \%$ with plasma-CTx, followed by CTx-plasma. 
a

C

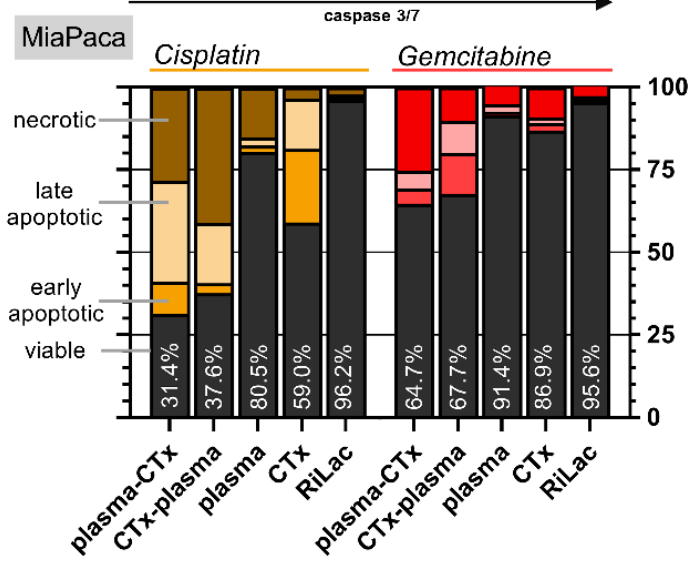

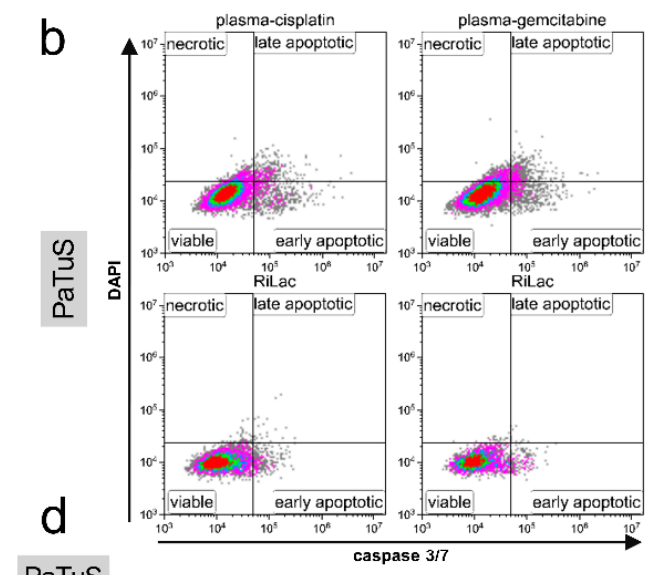

PaTuS

Cisplatin Gemcitabine

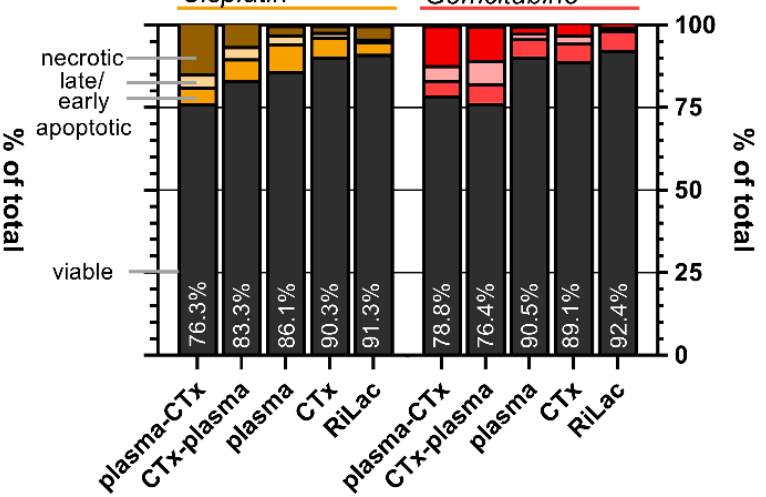

Figure 3. Combination of cisplatin and gemcitabine with physical plasma-conditioned Ringer's lactate induced toxicity in pancreatic cancer cells. (a,b) representative gating of viable (DAPI', caspase-), early apoptotic $\left(\mathrm{DAPI}^{-}\right.$, caspase $\left.^{+}\right)$, late apoptotic $\left(\mathrm{DAPI}^{+}\right.$, caspase $\left.^{+}\right)$and necrotic $\left(\mathrm{DAPI}^{+}\right.$, caspase $\left.{ }^{-}\right)$ MiaPaca (a) or PaTuS (b) cells at $48 \mathrm{~h}$ after exposure to plasma-CTx, CTx-plasma, or the corresponding Ringer's lactate control (RiLac); (c,d) quantification of the percentage of viable, early and late apoptotic, or necrotic cells at $48 \mathrm{~h}$ for (c) MiaPaca and (d) PaTuS pancreatic cancer cells. Data are presented as mean and are representatives out of three independent experiments.

\subsection{Combination Therapy Induced Morphological Alterations and Cell Cycle Arrest in Pancreatic Cancer Cells}

To further investigate the additive toxicity of the combination treatment on the pancreatic cancer cells, we performed quantitative high content image analysis on several cellular and morphological parameters (Figure 4a,b). To confirm cytotoxicity by fluorescence microscopy, exposing MiaPaca cells to cisplatin first and plasma-conditioned RiLac second led to a substantial and significant elevation of the percentage of dead cells (DAPI $/$ all cell events) (Figure 4c). For all other treatment regimens, cytotoxic responses were observed in tendency. Moreover, the total growth area of MiaPaca cells was also affected by the different treatment regimens at $48 \mathrm{~h}$ post initial exposure. In comparison to the RiLac control, both plasma-cisplatin and cisplatin-plasma and also cisplatin alone induced significant growth retardation (Figure $4 \mathrm{~d}$ ). A similar trend was observed when using gemcitabine. The altered cell growth features were accompanied by morphological changes. Individual MiaPaca cells exposed to cisplatin-plasma had an increased area per cell indicative of cellular swelling (Figure 4e). The swelling was also observed with the combination treatment using gemcitabine. As a second morphological feature, the individual cell's roundness significantly decreased in all combination treatment regimens in MiaPaca cells (Figure 4f). PaTuS cancer cells grow in larger aggregates, requiring different algorithms and quantitative techniques to investigate changes by microscopy. Cell death per growth area was calculated (DAPI ${ }^{+} /$area), and similarly to MiaPaca cells, cisplatin-plasma significantly increased toxicity in PaTuS cells (Figure 4g). Analyzing the growth characteristics of PaTuS cells following the different 
treatment regimens, significant declines were observed for plasma-cisplatin when compared to the RiLac control (Figure 4h).

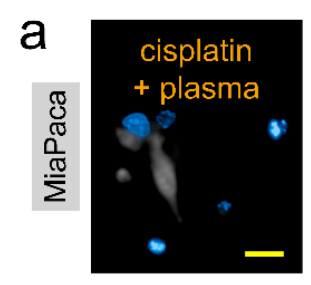

C MiaPaca $\square$ Cisplatin $\square$ Gemcitabine

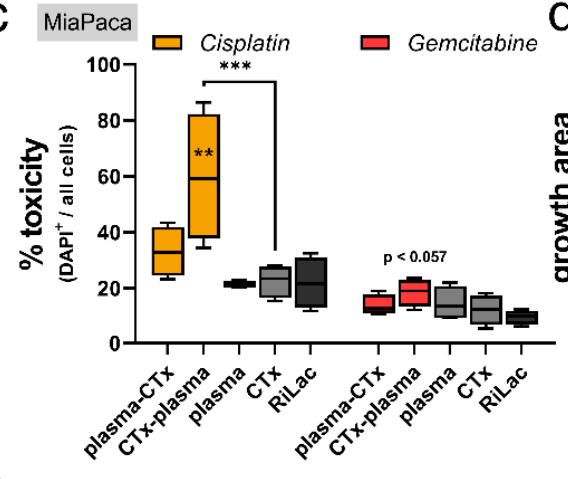

f

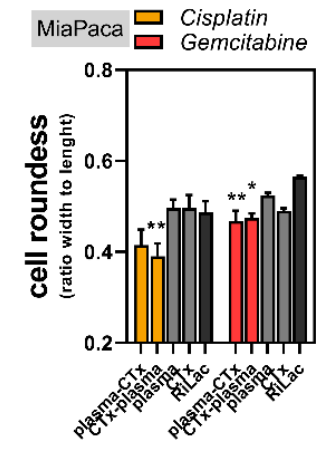

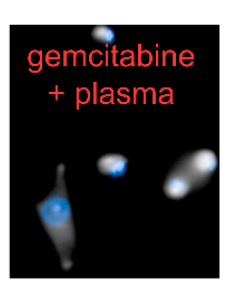

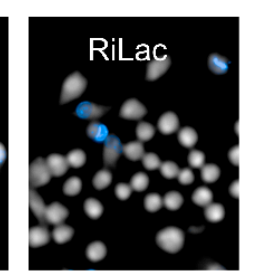

d MiaPaca
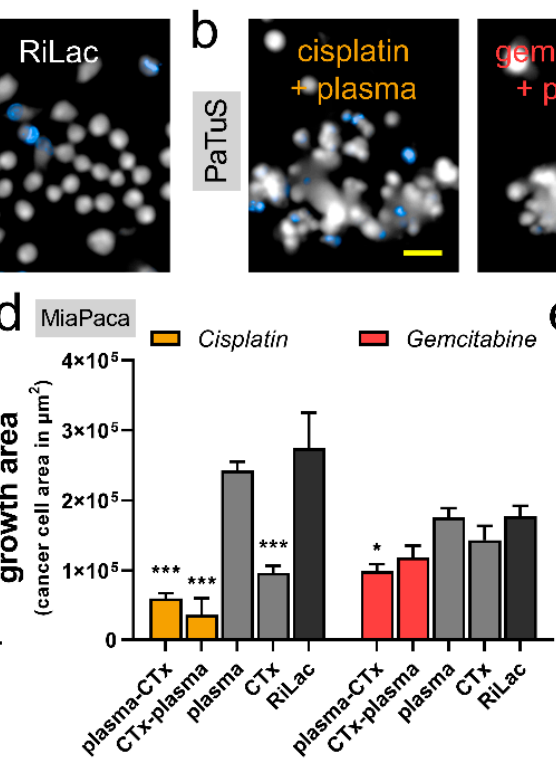
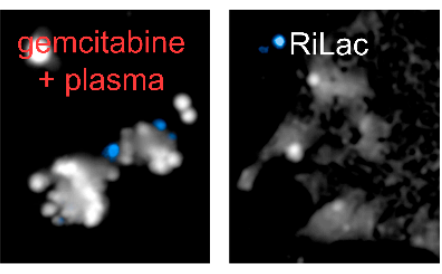

P MiaPaca $\square$ Cisplatin
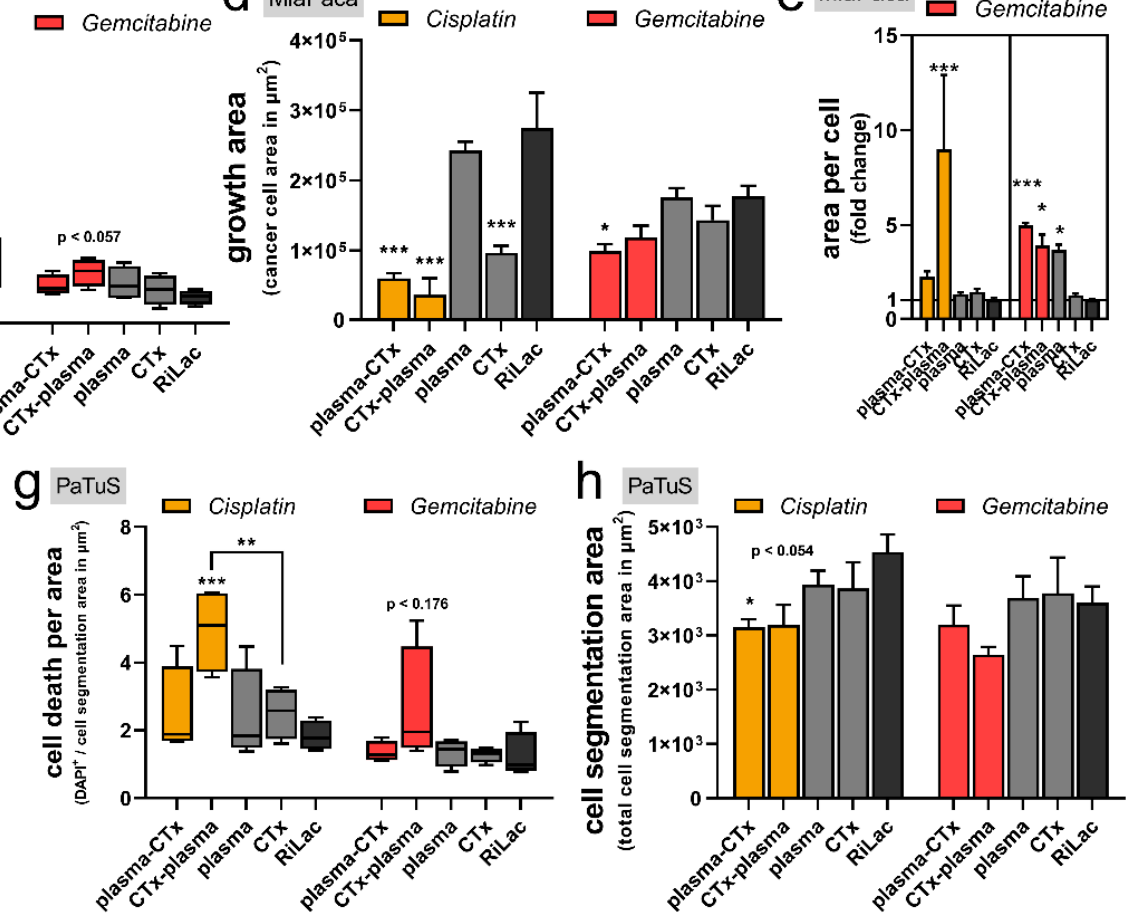

Figure 4. A combination of cisplatin and gemcitabine with physical plasma-conditioned Ringer's lactate was toxic, reduced the cancer cells' growth, and altered their morphology. (a) Representative images with the DAPI fluorescence channel and digital phase contrast of MiaPaca cells (scale-bar $=50 \mu \mathrm{m}$ ); (b) representative images with the DAPI fluorescence channel and digital phase contrast of PaTuS cell (scale-bar $=50 \mu \mathrm{m}) ;(\mathbf{c}-\mathbf{f})$ algorithm-based quantification of high-content imaging experiments showing (c) the toxicity (\% DAPI ${ }^{+}$events on all events), (d) the growth area (area of the pseudo-cytosolic digital phase contrast), (e) the area per cell, and (f) and roundness of MiaPaca pancreatic cancer cells; (g,h) algorithm-based quantification of ( $\mathrm{g}$ ) cell death $\left(\mathrm{DAPI}^{+}\right.$events per area) and (h) cell segmentation area of treated PaTuS cells. Imaging was performed at $48 \mathrm{~h}$ post initial exposure to the treatment liquids. Data are representative out of three independent experiments and are presented as (c,g) boxplot with their median \pm min and max, or $(\mathbf{e}-\mathbf{h})$ as mean + SEM. Statistical significance was calculated utilizing ANOVA.

Different growth properties and morphological alterations can be indicative of cellular senescence that is induced by an arrest of the cell cycle. To address this question, the content of nucleic acid inside the cells was quantified by flow cytometry, and the ratio of cells in the G2 over the G1 phase was calculated. The single treatment with physical plasma-conditioned RiLac introduced the most drastic changes in cell cycle arrest, except for the treatment with plasma-cisplatin in MiaPaca cells (Figure 5a-h). However, also drug mono treatment (except gemcitabine in MiaPaca cells) elevate the number of the cells stuck in the G2 fraction. In addition, all combination therapies, with the exception 
of gemcitabine-plasma in Miapaca cells, showed elevated cell cycle arrest compared to the RiLac control (Figure 5a-h).
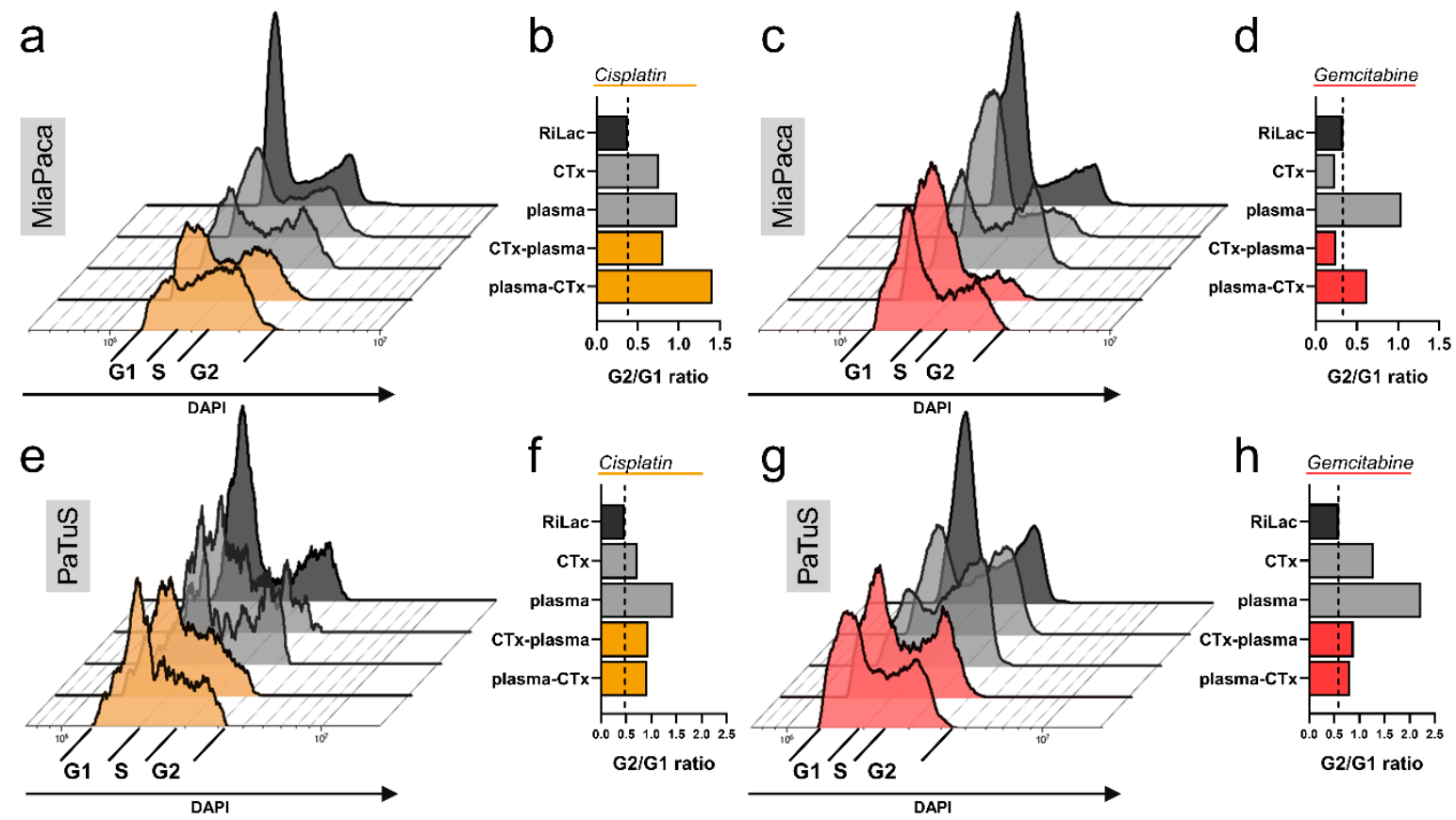

Figure 5. Plasma-conditioned Ringer's lactate induced cell cycle arrest in pancreatic cancer cells alone or in combination with chemotherapy. $(\mathbf{a}, \mathbf{c}, \mathbf{e}, \mathbf{g})$ Representative overlays of flow cytometry data of DNA content, showing the amount of treated pancreatic cancer cells in the G1, S, and G2 phase of the cell cycle; $(\mathbf{b}, \mathbf{d}, \mathbf{f}, \mathbf{h})$ quantification of cells in cell cycle arrest (G2/G1 ratio) for MiaPaca cells in treatment regimen with (b) cisplatin or (d) gemcitabine, as well as PaTuS cells after exposure to respective treatment regimens with (f) cisplatin and (h) gemcitabine $48 \mathrm{~h}$ post-treatment. Data show the mean and are representatives from three independent experiments.

\subsection{Drug Concentrations in Pancreatic Cancer Cells Changed in Combination Treatment as Analyzed Using} Mass Spectrometry

Next, we asked whether the exposition to plasma-conditioned RiLac alters the drug uptake and hence, the intracellular concentration of chemotherapeutic agents. To distinguish between acute effects, e.g., higher membrane penetrability (short-term), and prolonged effects, e.g., differences in expression or function of membrane transporter (long-term), the second treatment was performed with a latency of either $30 \mathrm{~min}$ or $24 \mathrm{~h}$. First, it was found that the intracellular drug levels were higher in MiaPaca compared to PaTuS cells (Figure 6a-d). Secondly, short-term concentrations were always higher than long-term concentrations. Third, intracellular levels of cisplatin were generally above those of gemcitabine. These findings directly correlate with the toxic effects that were observed in our cytotoxicity studies. Plasma-treated RiLac reduced intracellular drug levels in MiaPaca cells, regardless of the sequence of combination therapy. For PaTuS, a similar effect was observed in the short-term conditions, while long-term conditions showed roughly equal levels in mono and combination treatments. This points to altered drug uptake or efflux from pancreatic cancer cells exposed to plasma-treated RiLac. 


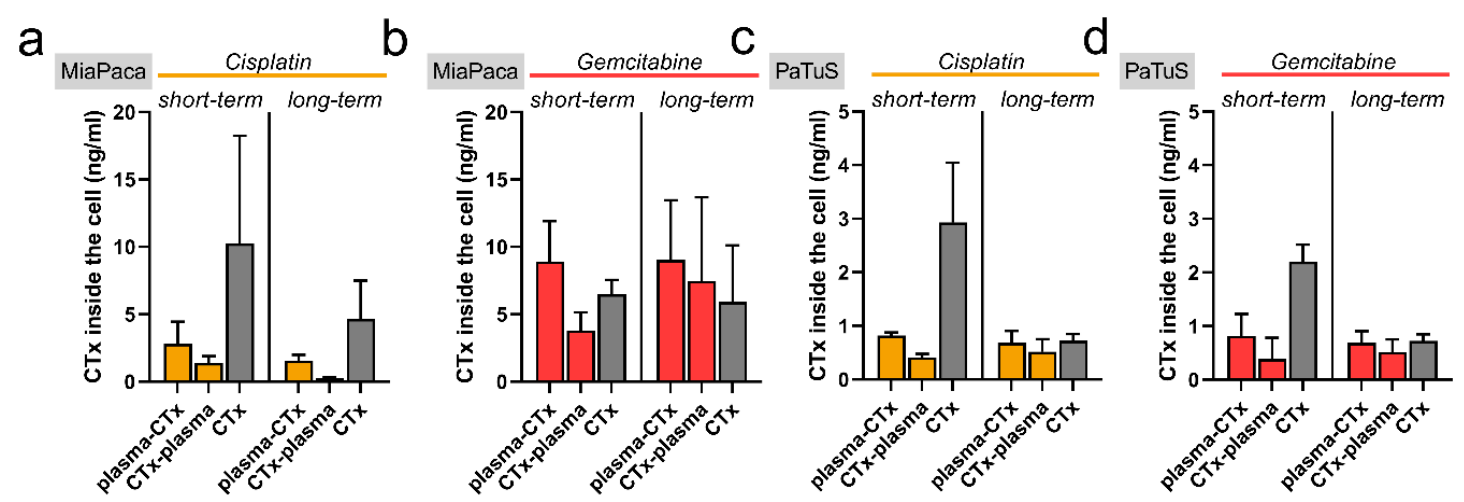

Figure 6. Initial cisplatin and gemcitabine uptake to pancreatic cancer cells were most effective in mono treatments but declined over time. (a,d) mass spectrometry quantification of the amount of the cisplatin and gemcitabine taken up to MiaPaca $(\mathbf{a}, \mathbf{b})$ and PaTuS $(\mathbf{c}, \mathbf{d})$ pancreatic cancer cells at short $(0.5 \mathrm{~h})$ and long-term $(24 \mathrm{~h})$. Data are mean + SEM from three independent experiments.

\subsection{Combination Treatment Abrogated Cancer Growth in an in Ovo 3D-Tumor Model}

To validate our findings in a more realistic model, MiaPaca and PaTuS pancreatic cancer cells were seeded on the chorion allantois membrane of fertilized chicken eggs (TUM-CAM model). This model allows for the growth of three-dimensional tumors (Figure 7a). While these tumors are void of immune cells, they become vascularized to form solid 3D-tumors. Treatment regimens were RiLac alone, plasma-conditioned RiLac, cisplatin alone, or cisplatin in combination with physical plasma-conditioned RiLac. All treatments were applied twice. It was waived to use gemcitabine in a combinational regimen on this in ovo model because cisplatin was identified to be the most promising candidate. The tumors were explanted $48 \mathrm{~h}$ after initial exposure to the liquids and weighted. For MiaPaca tumors, all treatment regimen significantly reduced the tumors mass compared to the RiLac control $(93.3 \pm 8.8 \mathrm{mg}$ ) (Figure $7 \mathrm{~b}$ ). Most tremendous changes were observed for the combination treatment with cisplatin and plasma that reduced the tumor mass to $52.9 \mathrm{mg}$ ( $\pm 5.6 \mathrm{mg}$ ), which was significantly lower than the cisplatin $(65.2 \pm 9.1 \mathrm{mg})$ and control $(76.8 \pm 6.6 \mathrm{mg})$ treatment (Figure $7 \mathrm{~b})$. Control PaTuS tumors were $105.3 \mathrm{mg}( \pm 38.6 \mathrm{mg})$. Combination treatment significantly retarded tumor growth to $49.8 \mathrm{mg}( \pm 17.9 \mathrm{mg})$. Cisplatin treatment gave $92.3 \mathrm{mg}( \pm 29.2 \mathrm{mg})$, while plasma-treated RiLac achieved a significant reduction to $62.1 \mathrm{mg}( \pm 28.6 \mathrm{mg}$ ) (Figure 7c). The ability to induce apoptosis is a crucial factor in the evaluation of oncologic strategies. Therefore, we examined the tumors by immunofluorescence for apoptosis by quantifying the amount of TUNEL ${ }^{+}$cells over all cells $\left(\mathrm{TUNEL}^{+} / \mathrm{DRAQ}^{+}\right.$). In MiaPaca tumors, spontaneous apoptosis was a rare event $(0.6 \pm 0.7 \%)$. Treatment with plasma $(19.2 \pm 9.7 \%)$ or cisplatin $(26.2 \pm 4.6 \%)$ significantly increased the rate of apoptotic cells. Combination treatment gave the strongest apoptotic response ( $38.8 \pm 12.1 \%)$ (Figure 7e). Next, we quantified the distance of apoptotic cells from the outer tumor layer and found a mean activity depth of $423 \mu \mathrm{m}( \pm 100 \mu \mathrm{m})$ with cisplatin (Figure 7f). However, both, plasma $(851 \pm 203.4 \mu \mathrm{m})$ and the combination treatment $(1003 \pm 222 \mu \mathrm{m})$ significantly showed significantly deeper penetration into the tissue (Figure 7f). In PaTuS tumors, spontaneous apoptosis was observed in $4.3 \%( \pm 5.9 \%)$ of the tumor cells. However, treatment with plasma $(20.0 \pm 5.8 \%)$ or cisplatin $(32.4 \pm 12.7 \%)$ alone, as well as the combination ( $37.2 \pm 17.9 \%$ ), significantly increased the rate of apoptosis (Figure $7 \mathrm{~h}$ ). By contrast, differences in penetration depth were not observed (Figure 7i). Furthermore, the percentage of proliferating $\left(\mathrm{Ki}-67^{+}\right)$cells was calculated. Tumors that received plasma-conditioned RiLac showed decreased proliferation in tendency, but differences were minor with all groups compared to the RiLac control (Supplementary Figure S1h,i). 

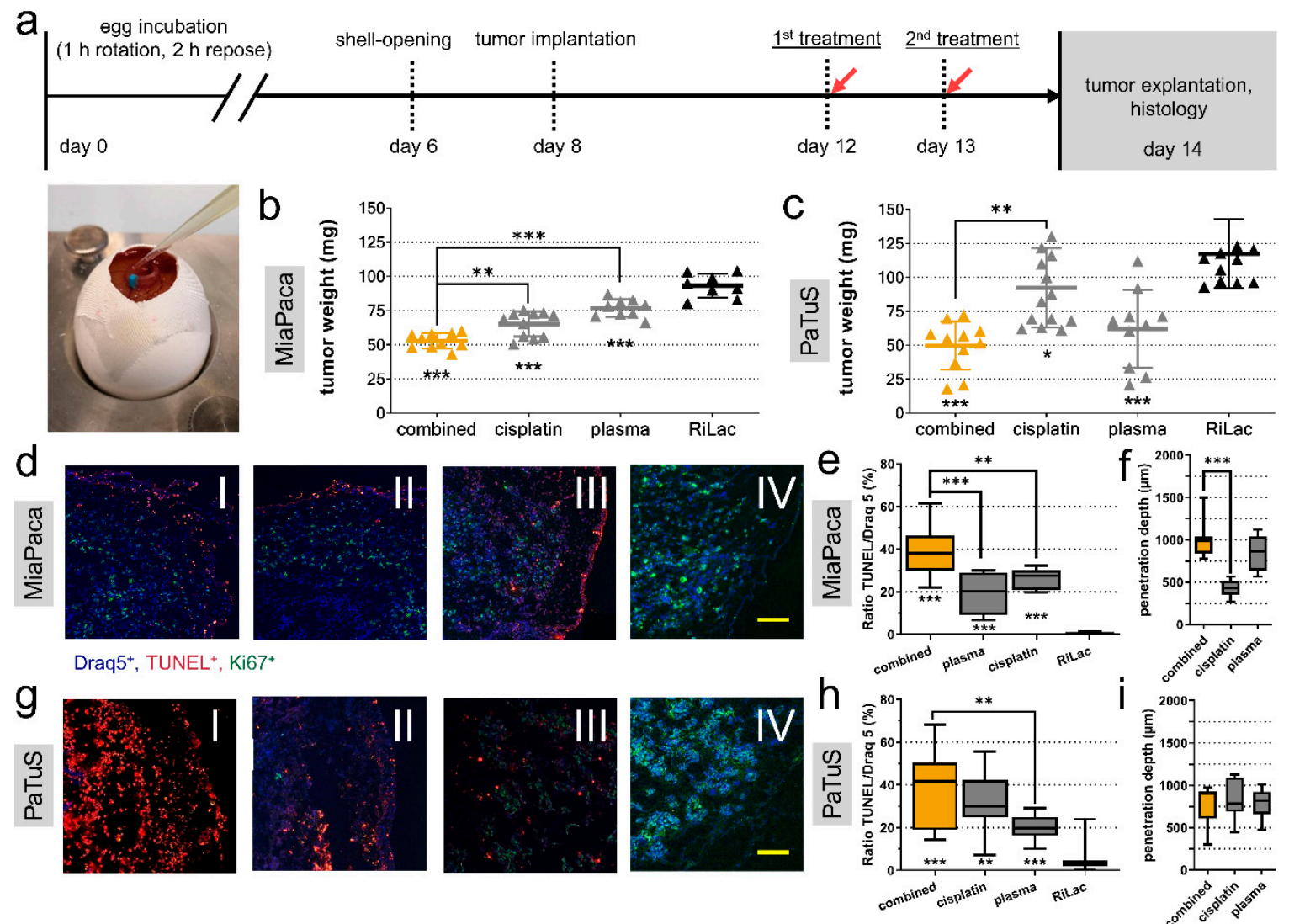

Figure 7. The combination of cisplatin with physical plasma-conditioned Ringer's lactate showed additive toxicity in three-dimensional tumors grown on chicken embryos. (a) Schematic overview of in ovo experiments and image of treatment with liquids of three-dimensional pancreatic tumors grown on the chorion-allantois membrane of fertilized chicken eggs (TUM-CAM); (b,c) tumor weight quantified at $48 \mathrm{~h}$ post-treatment with Ringer's lactate (RiLac), plasma-conditioned RiLac, cisplatin, or the combination of physical plasma-conditioned RiLac and cisplatin in MiaPaca (b) and PaTuS (c) tumors; $(\mathbf{d}, \mathbf{g})$ representative images of apoptotic MiaPaca (d) and PaTuS (g) cells (blue $=$ Draq5 ${ }^{+}$, red $=\mathrm{TUNEL}^{+}$, green $=\mathrm{Ki}^{+} 7^{+}$; scale-bar $=100 \mu \mathrm{m}$ ) in cryo-sections of the tumors exposed to the combination treatment (I), cisplatin (II), plasma-RiLac (III) and RiLac alone (IV); (e,h) algorithm-based quantitative image analysis of the percentage of apoptotic cells (TUNEL ${ }^{+} / \mathrm{Draq}^{+}$) in MiaPaca (e) and PaTuS (h) tumors; (f,i) penetration depth of the treatment regimen in MiaPaca (f) and PaTuS (i) tumors. Data are representative from two independent experiments with eight to thirteen eggs per group and show $(\mathbf{b}, \mathbf{c})$ individual values with their mean $\pm S D$, and $(\mathbf{e}, \mathbf{f}, \mathbf{h}, \mathbf{i})$ median and min to max. Statistical significance was calculated utilizing ANOVA.

\section{Discussion}

Most protocols in adjuvant, additive, and palliative CTx for PC are based on gemcitabine either as monotherapy or in combination with, for instance, capecitabine (ESPAC-4 [7]). Despite a better understanding of the pathogenesis of PC, the prognosis has improved only slightly in absolute terms. The best survival rates are observed in resected patients, with a median survival between 13 and 48 months, depending mainly on the tumor stage (i.e., T1/2 or T3/4) [7,8,29]. In metastatic non-resectable pancreatic cancer, median survival is between 5-7.5 months on palliative chemotherapy [30,31]. In this context, best survival rates (11 months) were observed using FOLFIRINOX, a gemcitabine-free scheme; however, its application is limited to those patients in good condition [32]. Therefore, gemcitabine is still the standard for most patients suffering from pancreatic PC. 
Nevertheless, and mainly due to the unsatisfactory results of chemotherapy, various pharmacological combination treatment schemes have been investigated in recent years, with usually only a slightly positive effect on survival coming at the costs of a significant increase in treatment-related toxicity [33]. One combination scheme involves gemcitabine and cisplatin, as used in biliary tract cancer [34], bladder carcinoma [35], or bronchial carcinoma [36]. This combination was also analyzed for PC and showed promising results with significantly improved six-month survival and better tumor response. However, no better overall survival could be demonstrated, and the incidence of CTx-related complications was also significantly increased compared to mono-treatment with gemcitabine alone [37].

After transfer into the cell, cisplatin forms adducts with the DNA, and as a consequence, a complex pathway of apoptotic and survival signaling is initiated [38]. Inactivation of cisplatin by intracellular scavengers (e.g., glutathione and metallothionein) is considered to be part of the drug resistance acquired during CTx [39]. Several studies investigated phytochemicals and their potential for sensitizing cancer cells for cisplatin-based CTx [40]. For example, shikonin, a product of a traditional Chinese medicinal plant, increased intracellular ROS concentration in vitro and in vivo and, therefore, enhanced cisplatin toxicity and notably raised selectivity [41].

It has been demonstrated multiple times that cold physical plasma applied either directly or via conditioned liquid acts as an anticancer agent in vitro [42-45]. Most studies highlight the prominent role of ROS and RNS, as the effects could be abolished by antioxidant scavengers [46]. Due to their high reactivity and usually short half-life, it is challenging to trace single reactive species in liquids or cells and estimate their contribution to biological effects [47-49]. In vivo, however, the previously promising in vitro results of plasma-conditioned liquids were reproduced in peritoneal metastatic tumors (gastric cancer [23], ovarian cancer [50], pancreatic cancer [22,51]), recently. These results raise hopes to integrate plasma medicine into modern, multimodal tumor therapies, mainly since the restoration of chemoresistance was observed in glioblastoma cells [18].

Surprisingly, there is little evidence of synergism between plasma treatment and CTx in pancreatic cancer yet. We previously demonstrated an additive effect with gemcitabine and plasma treatment in murine pancreatic cancer cells without additional harm to non-malignant fibroblasts [52]. In vivo, a significant tumor reduction in a murine, orthotopic pancreatic cancer model by the combination of plasma and gemcitabine was observed before [17]. It was also demonstrated that the sequence of administration of plasma and tegafur (a 5-FU prodrug) plays a decisive role between synergism and antagonism [53]. However, these studies were performed with direct plasma treatment and possibly included effects by gas, e.g., plasma-derived electromagnetic fields or UV-radiation. In our current study, we found an additive toxicity of plasma-conditioned RiLac with chemotherapy. This combination therapy was superior to mono-treatment in both cell lines investigated, as demonstrated by reduced metabolic activity and cell viability, and enhanced apoptosis and cell cycle arrest. Additionally, we demonstrated a significant improvement of the anti-cancer capacity of either cisplatin or plasma-conditioned RiLac in an in ovo TUM-CAM model by adding the other modality, respectively.

The first barrier clinically applied substances have to overcome is the cell membrane. ROS/RNS can penetrate or interact with this membrane, as well as use ubiquitous aquaporin channels, whereas cisplatin and gemcitabine enter the cell via different membrane transporters [54-57]. We hypothesized that plasma-conditioned liquid sensitizes the tumor cell for subsequent CTx via oxidative stress induction. By contrast, we found the intracellular concentration of cisplatin and gemcitabine being reduced in cells conditioned with plasma-treated RiLac. As a possible explanation, we hypothesize that cells exposed to plasma-conditioned RiLac experience oxidative stressed and became inactivated. Hence, the lower levels might have been due to reduced transporter activity in the short-term samples, and an increased portion of terminally dead cells (that nonetheless were still part of the cell pellet investigated by mass spectrometry) with the long-term samples. This hypothesis is supported by the reduction in the metabolic activity of the cells and by other hallmarks of cellular senescence. One of them is the cellular swelling (increased area per cell) that was observed to the greatest extent in our 
combinational regimen [58-60]. Moreover, cell cycle arrest was also observed in the groups that received the plasma-conditioned RiLac, similarly to our previous observations in colorectal cancer cells that had received plasma-conditioned saline [42].

With both cell lines investigated, gemcitabine was inferior compared to cisplatin in terms of cytotoxicity. The combination with plasma-conditioned RiLac was also less effective than that with cisplatin. This may be due to the mechanism of action of gemcitabine, which relies primarily on incorporation into the DNA. However, as we have been able to demonstrate, the plasma treatment leads to a significant increase in cells in the G2 phase, so Gemcitabine is correspondingly less incorporated. In contrast, plasma-derived radicals or cisplatin could reduce the antioxidant capacity of the cell so that the vice versa treatment was correspondingly more effective. This might point towards a more synergistic effect from plasma-conditioned RiLac and cisplatin.

The combination of plasma-conditioned RiLac and CTx was effective in vitro and in ovo. Applications of cytostatic drugs diluted in plasma-conditioned oxidative liquids (such as RiLac) therefore hold promising potential in, e.g., postoperative lavage or HIPEC. From our previous studies using intraperitoneal injections of plasma-conditioned sodium chloride or cell culture medium in mice, we know that plasma-conditioned liquids did not have any observable side effects [22,42]. Especially plasma-conditioned RiLac was previously demonstrated to have a potent anti-tumor capacity [61-63]. This was true in vitro and in vivo, and the lactate in RiLac was demonstrated being essential in mediating toxic effects [25,64]. These studies support our findings and hypothesis of such an oxidative liquid being suitable for future clinical applications in combination with standard chemotherapeutics (as cisplatin and gemcitabine) to reduce tumor burden in patients that suffer from, e.g., pancreatic cancer.

\section{Materials and Methods}

\subsection{Cell Lines and Cultivation}

Human pancreatic adenocarcinoma cell lines MiaPaca (MIA PaCa-2; ATCC, Manassas, VA, USA) and PaTuS (PaTu-8988s; DSMZ, Braunschweig, Germany) were used. Cells were maintained in cell culture medium (Dulbecco's modified Eagle's medium: DMEM GlutaMAX; Gibco ThermoFisher Scientific, Waltham, MA, USA) supplemented with $10 \%$ fetal calf serum, $100 \mathrm{U} / \mathrm{mL}$ of penicillin, and $100 \mu \mathrm{g} / \mathrm{mL}$ of streptomycin (all Sigma, Steinheim, Germany). The confluence of cells was controlled via microscopy, and subculturing was performed twice a week, while the passages of the cells were kept below ten. Incubation took place in a cell culture incubator (Binder, Tuttlingen, Germany) at $37^{\circ} \mathrm{C}$ and $5 \% \mathrm{CO}_{2}$ under humidified conditions. Possible mycoplasma contamination of the cell culture was excluded regularly. For the experimental in vitro procedures, the cells were counted using an acoustic-focussing flow cytometer (Attune NxT, ThermoFisher Scientific) and added at a concentration of $1 \times 10^{4}$ cells in $100 \mu \mathrm{L}$ medium per well in 96-well plates. Alternatively, $1 \times 10^{5}$ cells were seeded in $1000 \mu \mathrm{L}$ medium in 24-well flatbottom plates (Eppendorf, Hamburg, Germany) for flow cytometry experiments. The therapeutic liquids were scaled up $10 \times$ in this setting. Both plate types provide a rim that can be filled with double-distilled water, to prevent excessive evaporation of the cell culture medium in the edge wells.

\subsection{Physical Plasma and Chemotherapeutic Agents}

Cold physical plasma was generated by the atmospheric pressure argon-plasma jet kINPen (neoplas tools $\mathrm{GmbH}$, Greifswald, Germany), operated at 1.9-3.2 W and 1.1 MHz, and CE-certified as a medical device class IIa in 2013 [65]. The plasma jet consists of a power supply unit and a handpiece. The latter contains a rod-shaped electrode that excites the argon gas (purity: 99.999\%; Air Liquide, Paris, France). Outwardly, the device is shielded by a dielectric capillary. For the plasma-treatment of Ringer's lactate (RiLac; Hartmann B. Braun, Melsungen, Germany), $100 \mu \mathrm{L}$ of the liquid was exposed to the plasma of the device running at two standard liters per minute $(2 \mathrm{slm})$. The visible plasma effluent did not 
directly discharge to the liquid. The distance of the liquid surface to the nozzle $(9 \mathrm{~mm})$ as well as treatment times regulated with high precision utilizing a computer-controlled $x y z$-table (CNC step, Geldern, Germany). Immediately after the plasma-conditioning, the plasma-RiLac was transferred to the plate, harboring the pancreatic cancer cells with just priorily aspirated cell culture supernatant. Treatment agents were left for $30 \mathrm{~min}$ before replacing them with fresh culture medium. For the detection of $\mathrm{H}_{2} \mathrm{O}_{2}$ in the RiLac, the Amplex UltraRed Assay (ThermoFisher Scientific), and for the detection of nitrate in nitrite, the Griess assay (Cayman Chemical, Ann Arbor, MI, USA) was utilized according to the manufacturers' instructions. The chemotherapeutic agents cisplatin $(1 \mathrm{mg} / \mathrm{mL}$, Teva, Petach Tikwa, Israel) and gemcitabine $(1 \mathrm{mg} / \mathrm{mL}$, provided by the University Pharmacy Greifswald, Greifswald, Germany) were stored for a maximum of four weeks at room temperature and protected from light. Following long plasma exposure times, the amount of evaporated liquid was measured via a precision balance (Sartorius, Göttingen, Germany) and was supplemented with double distilled water. Standard dilutions (Table 1) were done in RiLac, and the cells were exposed to the drugs in a similar regimen as for the plasma-RiLac. Oxidation of the hydroxyl radical and peroxynitrite anion indicator hydroxyphenyl fluorescein (HPF, ThermoFisher Scientific) was measured immediately after the treatment of $5 \mu \mathrm{M}$ of the probe after $120 \mathrm{~s}$ of plasma-exposure at the $\lambda_{\mathrm{ex}}=460-490 \mathrm{~nm}$ and $\lambda_{\mathrm{em}}=500-550 \mathrm{~nm}$ fluorescence channel of a high content imager (Operetta CLS, PerkinElmer, Hamburg, Germany). Measurements of the $\mathrm{pH}$ were performed with a $\mathrm{pH}$ meter (Mettler Toledo, Columbus, $\mathrm{OH}$, USA).

\subsection{Metabolic Activity Detection}

To asses the metabolic activity of the cancer cells after treatment, they were exposed to 7-hydroxy-3H-phenoxazin-3-on-10-oxid (resazurin, Alfa Aesar, Haverhill, MA, USA) at $20 \mathrm{~h}$. The dye can be metabolized by viable cells to generate fluorescent resorufin. Fluorescence was measured after $4 \mathrm{~h}$ utilizing a multiplate reader (Tecan F200, Tecan, Männedorf, Switzerland) at $\lambda_{\mathrm{ex}}=560 \mathrm{~nm}$ and $\lambda_{\mathrm{em}}=590 \mathrm{~nm}$. The $\mathrm{H}_{2} \mathrm{O}_{2}$ scavenging enzyme catalase (Sigma, Steinheim, Germany) was added at concentrations of $20 \mu \mathrm{g} / \mathrm{mL}$ in some control experiments prior to addition of plasma-conditioned liquid.

\subsection{Flow cytometry}

After incubation, cells were washed with phosphate-buffered saline (PBS; PAN Biotech, Aidenbach, Germany) and detached with accutase (BioLegend, San Diego, CA, USA) containing DAPI (BioLegend) and Caspase 3/7 Green Detection Reagent (ThermoFisher Scientific). The cells were stained for $30 \mathrm{~min}$ at $37^{\circ} \mathrm{C}$ before washing twice with PBS. Data acquisition was performed with a CytoFLEX $S$ flow cytometer (Beckman-Coulter, Brea, CA, USA). For each of the replicates, 10,000 single cells were acquired and analyzed using Kaluza 2.1 analysis software (Beckman-Coulter). For cell cycle analysis, cells were harvested and then incubated with ice-cold ethanol at $-20^{\circ} \mathrm{C}$ for $1 \mathrm{~h}$. After washing and staining with DAPI, flow cytometry data were analyzed for cell cycle phases using the Michael $\mathrm{H}$. Fox algorithm that is provided within the Kaluza software.

\subsection{High Content Imaging}

Imaging was performed using a high content imager (Operetta CLS). The device operates a high-speed motorized table. Images were acquired using a $20 \mathrm{x}$ air objective (NA $=0.4$; Zeiss, Jena, Germany) and a 16-bit sCMOS camera with laser-based autofocus. Cells were stained with DAPI in images in the brightfield, digital-phase contrast (DPC), and the $\lambda_{\mathrm{ex}}=535-585 \mathrm{~nm}$ and $\lambda_{\text {em }}=430-500 \mathrm{~nm}$ fluorescence channels were acquired. Image acquisition settings were kept constant. For each independent experiment, four technical replicates with a total of 36 fields of views per condition and experiment were imaged. For the quantification of cell counts, cell area, and morphology, an algorithm-based analysis was performed using Harmony 4.9 image acquisition and quantification software (PerkinElmer, Hamburg, Germany) after segmenting individual cells via their pseudo-cytosolic DPC signal. 


\subsection{Quantitative Assay for Cisplatin and Gemcitabine}

Cisplatin and gemcitabine were quantified in cell pellets after lysis and homogenization of the cells and protein precipitation by adding $500 \mu \mathrm{L}$ acetonitrile and subsequent centrifugation for $5 \mathrm{~min}$ at $14,000 \mathrm{~g}$ and $4{ }^{\circ} \mathrm{C}$. Ten microliters of the clear supernatant were subjected to liquid chromatography-tandem mass spectrometry (LC-MS/MS) analysis. The analysis was performed with the Agilent 1290 series HPLC system (Agilent Technologies, Waldbronn, Germany) coupled with the QTRAP5500 mass spectrometer (Sciex, Darmstadt, Germany). Chromatography was performed using the analytical column Atlantis ${ }^{\circledR}$ HILIC Silica column, $2.1 \times 100 \mathrm{~mm}$ (Waters, Milford, CT, USA) by gradient elution with acetonitrile (A) and $0.1 \%$ formic acid (B) as mobile phases at a flow rate of $250 \mu \mathrm{L} / \mathrm{min}$. The applied gradient was as follows: 0-1 $\mathrm{min}, 99 \% \mathrm{~A} ; 1-1.1 \mathrm{~min}, 30 \% \mathrm{~A} ; 1.1-3 \mathrm{~min} 30 \% \mathrm{~A}$; 3-3.1 $\mathrm{min} 99 \% \mathrm{~A}$; and 3.1-8 min 99\% A. The MS/MS analysis was done in the positive multiple reaction monitoring mode by considering the following mass-to-charge transitions (and collision energies): 302.0/246.0 (12 eV), 302.0/266.0 (20 eV) and 302.0/210.0 (36 eV) for cisplatin and 246.2/111.6 (23 eV), $246.2 / 95(60 \mathrm{eV})$ and 246.2/69 (55 eV) for gemcitabine. The analytical range of the quantitative method for both compounds was between 1-100 ng/mL. During the period of sample analysis, the accuracy of the method was $\pm 15 \%$ (relative error of the nominal values).

\subsection{Tumor-Chorion-Allantoic Membrane Model (TUM-CAM)}

The chorion-allantois membrane tumor model (TUM-CAM) was performed as described in previous studies [66,67]. Briefly, pathogen-free eggs (Valo BioMedia, Osterholz-Scharmbeck, Germany) were incubated for one week at a specialized egg incubator with turning functions (Hemel, Verl, Germany) at $37.5^{\circ} \mathrm{C}$ and $65 \%$ humidity. On day eight, the eggshells were carefully opened, and a cell suspension (containing $2 \times 10^{6}$ cells in $50 \mu \mathrm{L}$ matrigel extracellular matrix components; Corning, New York, NY, USA) was added to a sterile silicone ring that was placed on the chorion-allantois membrane (CAM). After a further incubation period of four days, solid tumors with blood vessels sprouting from the CAM have formed inside the ring. On day 12, the treatment was performed. For this, the eggs were randomly assigned to groups and received (I) control RiLac, (II) plasma-conditioned RiLac, (III) cisplatin, or (IV) a combination of plasma-RiLac and cisplatin. RiLac volume was $100 \mu \mathrm{L}$ of, and the concentrations of the drugs and plasma are given in Table 1. Following treatment, the eggs were restored in the breeder, and on the next day, treatment was repeated. On day 14, tumors were excised and cryo-conservated in liquid nitrogen $\left(-196{ }^{\circ} \mathrm{C}\right)$ embedded in freezing medium (Tissue-Tek O.C.T., Sakura Europe, Alphen aan den Rijn, Netherlands).

\subsection{Histology}

Ultra-thin sections $(5 \mu \mathrm{m})$ were cut vertically and mounted on microscope slides. Nuclei were counterstained using Draq5 (BioLegend). Apoptotic cells were labeled using the TUNEL assay (In situ cell death detection kit, TMR red; Merck, Darmstadt, Germany) according to manufacturer's specifications. Proliferating cells were labeled using an anti-Ki67 monoclonal antibody (primary antibody: rabbit anti-Ki67; Bethyl Laboratories, Montgomery, TX, USA) that was marked using a fluorescently labeled secondary antibody (donkey anti-rabbit IgG Brilliant Violet 421; BioLegend)). Microscopy slides were examined using a Keyence BZ-9000 fluorescence microscope (Keyence, Frankfurt, Germany). Using the software dynamic cell count (BZ-II Analyser, Keyence, Frankfurt, Germany), the ratio of TUNEL positive and negative cells (TUNEL ${ }^{+} / \mathrm{Draq}^{+}{ }^{+} \mathrm{vs} \mathrm{TUNEL}^{-} / \mathrm{Draq}^{+}$) $\mathrm{was}^{2}$ determined [43].

\subsection{Statistical Analysis}

Statistical analysis was performed using Prism 8.3 (GraphPad Software, La Jolla, CA, USA). For statistical comparison between different groups, one-way or two-way analysis of variance (ANOVA II) with Dunnet's post-testing was applied. The levels of significance are displayed as asterisks 
in the figures $\left({ }^{*}, * *\right.$, or ${ }^{* * *}$ for the $p$-values $<0.05,<0.01$, or $<0.001$, respectively). All experiments were performed at least in three independent runs, and detailed information about the specific number of replicates as well es the presentation of the data (mean/median, min-max, SD/SEM) is given in the figure captions.

\section{Conclusions}

The therapy of pancreatic cancer remains challenging, mainly due to its aggressive and infiltrating growth, e.g., to the peritoneal cavity. Our data suggest that standard anti-cancer chemotherapies of this cavity may benefit from a novel combination therapy using pro-oxidative Ringer's lactate generated via cold physical plasma. Our approach is elegant and clinically relevant because both the Ringer's lactate as well as the kINPen used in this study are clinically certified products theoretically ready to be used. Further studies are needed using, for instance, tumor materials from patients ex vivo, or launching individual therapy trials with patients in experimental settings.

Supplementary Materials: The following are available online at http://www.mdpi.com/2072-6694/12/1/123/s1, Figure S1: Additional information.

Author Contributions: Conceptualization: K.-R.L., E.F. and S.B.; Data curation: K.-R.L., E.F., M.H., and S.O.; Formal analysis: K.-R.L., E.F., M.H., S.O., and S.B.; Funding acquisition: C.-D.H., L.-I.P., and S.B.; Investigation: K.-R.L., E.F., M.H., and S.O.; Methodology: K.-R.L., E.F., S.O., and S.B.; Project administration: K.-R.L., E.F., C.-D.H., L.-I.P., and S.B.; Resources: C.D.W., L.-I.P., and S.B.; Software: E.F.; Supervision: K.-R.L., C.-D.H., L.-I.P., and S.B.; Validation: E.F., M.H., and S.O.; Visualization: E.F. and S.B.; Writing-original draft: E.F. and S.B.; Writing-review and editing: K.-R.L., E.F., M.H., S.O., C.-D.H., L.-I.P., and S.B. All authors have read and agreed to the published version of the manuscript.

Funding: Sander Bekeschus and Eric Freund are supported by the Federal German Ministry of Education and Research (grant number: 03Z22DN11).

Acknowledgments: We thankfully acknowledge technical support by Felix Nießner and Antje Janetzko.

Conflicts of Interest: The authors declare no conflict of interest.

\section{References}

1. Ferlay, J.; Colombet, M.; Soerjomataram, I.; Mathers, C.; Parkin, D.M.; Pineros, M.; Znaor, A.; Bray, F. Estimating the global cancer incidence and mortality in 2018: GLOBOCAN sources and methods. Int. J. Cancer 2019, 144, 1941-1953. [CrossRef] [PubMed]

2. Siegel, R.L.; Miller, K.D.; Jemal, A. Cancer statistics, 2019. CA Cancer J. Clin. 2019, 69, 7-34. [CrossRef] [PubMed]

3. Kamisawa, T.; Wood, L.D.; Itoi, T.; Takaori, K. Pancreatic cancer. Lancet 2016, 388, 73-85. [CrossRef]

4. Kikuyama, M.; Kamisawa, T.; Kuruma, S.; Chiba, K.; Kawaguchi, S.; Terada, S.; Satoh, T. Early Diagnosis to Improve the Poor Prognosis of Pancreatic Cancer. Cancers 2018, 10, 48. [CrossRef]

5. Soriano, A.; Castells, A.; Ayuso, C.; Ayuso, J.R.; de Caralt, M.T.; Gines, M.A.; Real, M.I.; Gilabert, R.; Quinto, L.; Trilla, A.; et al. Preoperative staging and tumor resectability assessment of pancreatic cancer: Prospective study comparing endoscopic ultrasonography, helical computed tomography, magnetic resonance imaging, and angiography. Am. J. Gastroenterol. 2004, 99, 492-501. [CrossRef]

6. Strobel, O.; Neoptolemos, J.; Jäger, D.; Büchler, M.W. Optimizing the outcomes of pancreatic cancer surgery. Nat. Rev. Clin. Oncol. 2019, 16, 11-26. [CrossRef]

7. Neoptolemos, J.P.; Palmer, D.H.; Ghaneh, P.; Psarelli, E.E.; Valle, J.W.; Halloran, C.M.; Faluyi, O.; O’Reilly, D.A.; Cunningham, D.; Wadsley, J.; et al. Comparison of adjuvant gemcitabine and capecitabine with gemcitabine monotherapy in patients with resected pancreatic cancer (ESPAC-4): a multicentre, open-label, randomised, phase 3 trial. Lancet 2017, 389, 1011-1024. [CrossRef]

8. Oettle, H.; Neuhaus, P.; Hochhaus, A.; Hartmann, J.T.; Gellert, K.; Ridwelski, K.; Niedergethmann, M.; Zülke, C.; Fahlke, J.; Arning, M.B.; et al. Adjuvant chemotherapy with gemcitabine and long-term outcomes among patients with resected pancreatic cancer: The conko-001 randomized trial. JAMA 2013, 310, 1473-1481. [CrossRef] 
9. Rahib, L.; Smith, B.D.; Aizenberg, R.; Rosenzweig, A.B.; Fleshman, J.M.; Matrisian, L.M. Projecting cancer incidence and deaths to 2030: The unexpected burden of thyroid, liver, and pancreas cancers in the United States. Cancer Res. 2014, 74, 2913-2921. [CrossRef]

10. Trachootham, D.; Alexandre, J.; Huang, P. Targeting cancer cells by ROS-mediated mechanisms: A radical therapeutic approach? Nat. Rev. Drug Discov. 2009, 8, 579-591. [CrossRef]

11. Pinto, A.; Mace, Y.; Drouet, F.; Bony, E.; Boidot, R.; Draoui, N.; Lobysheva, I.; Corbet, C.; Polet, F.; Martherus, R.; et al. A new ER-specific photosensitizer unravels (1)O2-driven protein oxidation and inhibition of deubiquitinases as a generic mechanism for cancer PDT. Oncogene 2016, 35, 3976-3985. [CrossRef] [PubMed]

12. Pinto, A.; Pocard, M. Photodynamic therapy and photothermal therapy for the treatment of peritoneal metastasis: a systematic review. Pleura Peritoneum 2018, 3, 20180124. [CrossRef] [PubMed]

13. von Woedtke, T.; Schmidt, A.; Bekeschus, S.; Wende, K.; Weltmann, K.D. Plasma Medicine: A Field of Applied Redox Biology. In Vivo 2019, 33, 1011-1026. [CrossRef] [PubMed]

14. Privat-Maldonado, A.; Gorbanev, Y.; Dewilde, S.; Smits, E.; Bogaerts, A. Reduction of Human Glioblastoma Spheroids Using Cold Atmospheric Plasma: The Combined Effect of Short- and Long-Lived Reactive Species. Cancers (Basel) 2018, 10, 394. [CrossRef]

15. Kaushik, N.K.; Kaushik, N.; Adhikari, M.; Ghimire, B.; Linh, N.N.; Mishra, Y.K.; Lee, S.J.; Choi, E.H. Preventing the Solid Cancer Progression via Release of Anticancer-Cytokines in Co-Culture with Cold Plasma-Stimulated Macrophages. Cancers (Basel) 2019, 11, 842. [CrossRef]

16. Bekeschus, S.; Lippert, M.; Diepold, K.; Chiosis, G.; Seufferlein, T.; Azoitei, N. Physical plasma-triggered ROS induces tumor cell death upon cleavage of HSP90 chaperone. Sci. Rep. 2019, 9, 4112. [CrossRef]

17. Brulle, L.; Vandamme, M.; Ries, D.; Martel, E.; Robert, E.; Lerondel, S.; Trichet, V.; Richard, S.; Pouvesle, J.M.; Le Pape, A. Effects of a non thermal plasma treatment alone or in combination with gemcitabine in a MIA PaCa2-luc orthotopic pancreatic carcinoma model. Plos ONE 2012, 7, e52653. [CrossRef]

18. Koritzer, J.; Boxhammer, V.; Schafer, A.; Shimizu, T.; Klampfl, T.G.; Li, Y.F.; Welz, C.; Schwenk-Zieger, S.; Morfill, G.E.; Zimmermann, J.L.; et al. Restoration of sensitivity in chemo-resistant glioma cells by cold atmospheric plasma. PLoS ONE 2013, 8, e64498. [CrossRef]

19. Conway, G.E.; Casey, A.; Milosavljevic, V.; Liu, Y.; Howe, O.; Cullen, P.J.; Curtin, J.F. Non-thermal atmospheric plasma induces ROS-independent cell death in U373MG glioma cells and augments the cytotoxicity of temozolomide. Br. J. Cancer 2016, 114, 435-443. [CrossRef]

20. Sagwal, S.K.; Pasqual-Melo, G.; Bodnar, Y.; Gandhirajan, R.K.; Bekeschus, S. Combination of chemotherapy and physical plasma elicits melanoma cell death via upregulation of SLC22A16. Cell Death Dis. 2018, 9, 1179. [CrossRef]

21. Kaushik, N.K.; Ghimire, B.; Li, Y.; Adhikari, M.; Veerana, M.; Kaushik, N.; Jha, N.; Adhikari, B.; Lee, S.J.; Masur, K.; et al. Biological and medical application of plasma-activated media, water and solutions. Biol. Chem. 2018, 400, 39-62. [CrossRef] [PubMed]

22. Liedtke, K.R.; Bekeschus, S.; Kaeding, A.; Hackbarth, C.; Kuehn, J.P.; Heidecke, C.D.; von Bernstorff, W.; von Woedtke, T.; Partecke, L.I. Non-thermal plasma-treated solution demonstrates antitumor activity against pancreatic cancer cells in vitro and in vivo. Sci. Rep. 2017, 7, 8319. [CrossRef] [PubMed]

23. Takeda, S.; Yamada, S.; Hattori, N.; Nakamura, K.; Tanaka, H.; Kajiyama, H.; Kanda, M.; Kobayashi, D.; Tanaka, C.; Fujii, T.; et al. Intraperitoneal Administration of Plasma-Activated Medium: Proposal of a Novel Treatment Option for Peritoneal Metastasis From Gastric Cancer. Ann. Surg. Oncol. 2017, 24, 1188-1194. [CrossRef] [PubMed]

24. Freund, E.; Liedtke, K.R.; Gebbe, R.; Heidecke, A.K.; Partecke, L.-I.; Bekeschus, S. In Vitro Anticancer Efficacy of Six Different Clinically Approved Types of Liquids Exposed to Physical Plasma. IEEE Trans. Radiat. Plasma Med. Sci. 2019, 3, 588-596. [CrossRef]

25. Tanaka, H.; Nakamura, K.; Mizuno, M.; Ishikawa, K.; Takeda, K.; Kajiyama, H.; Utsumi, F.; Kikkawa, F.; Hori, M. Non-thermal atmospheric pressure plasma activates lactate in Ringer's solution for anti-tumor effects. Sci. Rep. 2016, 6, 36282. [CrossRef]

26. Roviello, F.; Pinto, E.; Corso, G.; Pedrazzani, C.; Caruso, S.; Filippeschi, M.; Petrioli, R.; Marsili, S.; Mazzei, M.A.; Marrelli, D. Safety and potential benefit of hyperthermic intraperitoneal chemotherapy (HIPEC) in peritoneal carcinomatosis from primary or recurrent ovarian cancer. J. Surg. Oncol. 2010, 102, 663-670. [CrossRef] 
27. Votanopoulos, K.I.; Newman, N.A.; Russell, G.; Ihemelandu, C.; Shen, P.; Stewart, J.H.; Levine, E.A. Outcomes of Cytoreductive Surgery (CRS) with hyperthermic intraperitoneal chemotherapy (HIPEC) in patients older than 70 years; survival benefit at considerable morbidity and mortality. Ann. Surg. Oncol. 2013, 20, 3497-3503. [CrossRef]

28. Pace, R.T.; Burg, K.J. Toxic effects of resazurin on cell cultures. Cytotechnology 2015, 67, 13-17. [CrossRef]

29. Neoptolemos, J.P.; Dunn, J.A.; Stocken, D.D.; Almond, J.; Link, K.; Beger, H.; Bassi, C.; Falconi, M.; Pederzoli, P.; Dervenis, C.; et al. Adjuvant chemoradiotherapy and chemotherapy in resectable pancreatic cancer: A randomised controlled trial. Lancet 2001, 358, 1576-1585. [CrossRef]

30. Wang, Y.; Hu, G.F.; Zhang, Q.Q.; Tang, N.; Guo, J.; Liu, L.Y.; Han, X.; Wang, X.; Wang, Z.H. Efficacy and safety of gemcitabine plus erlotinib for locally advanced or metastatic pancreatic cancer: a systematic review and meta-analysis. Drug Des. Devel. 2016, 10, 1961-1972. [CrossRef]

31. Hubner, R.A.; Worsnop, F.; Cunningham, D.; Chau, I. Gemcitabine plus capecitabine in unselected patients with advanced pancreatic cancer. Pancreas 2013, 42, 511-515. [CrossRef]

32. Conroy, T.; Desseigne, F.; Ychou, M.; Bouche, O.; Guimbaud, R.; Becouarn, Y.; Adenis, A.; Raoul, J.L.; Gourgou-Bourgade, S.; de la Fouchardiere, C.; et al. FOLFIRINOX versus gemcitabine for metastatic pancreatic cancer. N. Engl. J. Med. 2011, 364, 1817-1825. [CrossRef] [PubMed]

33. Ciliberto, D.; Botta, C.; Correale, P.; Rossi, M.; Caraglia, M.; Tassone, P.; Tagliaferri, P. Role of gemcitabine-based combination therapy in the management of advanced pancreatic cancer: a meta-analysis of randomised trials. Eur. J. Cancer 2013, 49, 593-603. [CrossRef] [PubMed]

34. Valle, J.; Wasan, H.; Palmer, D.H.; Cunningham, D.; Anthoney, A.; Maraveyas, A.; Madhusudan, S.; Iveson, T.; Hughes, S.; Pereira, S.P.; et al. Cisplatin plus Gemcitabine versus Gemcitabine for Biliary Tract Cancer. N. Engl. J. Med. 2010, 362, 1273-1281. [CrossRef] [PubMed]

35. Galsky, M.D.; Pal, S.K.; Chowdhury, S.; Harshman, L.C.; Crabb, S.J.; Wong, Y.N.; Yu, E.Y.; Powles, T.; Moshier, E.L.; Ladoire, S.; et al. Comparative effectiveness of gemcitabine plus cisplatin versus methotrexate, vinblastine, doxorubicin, plus cisplatin as neoadjuvant therapy for muscle-invasive bladder cancer. Cancer 2015, 121, 2586-2593. [CrossRef]

36. Schiller, J.H.; Harrington, D.; Belani, C.P.; Langer, C.; Sandler, A.; Krook, J.; Zhu, J.; Johnson, D.H. Comparison of Four Chemotherapy Regimens for Advanced Non-Small-Cell Lung Cancer. N. Engl. J. Med. 2002, 346, 92-98. [CrossRef]

37. Ouyang, G.; Liu, Z.; Huang, S.; Li, Q.; Xiong, L.; Miao, X.; Wen, Y. Gemcitabine plus cisplatin versus gemcitabine alone in the treatment of pancreatic cancer: A meta-analysis. World J. Surg. Oncol. 2016, 14, 59. [CrossRef]

38. Siddik, Z.H. Cisplatin: Mode of cytotoxic action and molecular basis of resistance. Oncogene 2003, 22, 7265-7279. [CrossRef]

39. Amable, L. Cisplatin resistance and opportunities for precision medicine. Pharm. Res. 2016, 106, $27-36$. [CrossRef]

40. Sun, C.Y.; Zhang, Q.Y.; Zheng, G.J.; Feng, B. Phytochemicals: Current strategy to sensitize cancer cells to cisplatin. Biomed. Pharm. 2019, 110, 518-527. [CrossRef]

41. He, G.; He, G.; Zhou, R.; Pi, Z.; Zhu, T.; Jiang, L.; Xie, Y. Enhancement of cisplatin-induced colon cancer cells apoptosis by shikonin, a natural inducer of ROS in vitro and in vivo. Biochem. Biophys. Res. Commun. 2016, 469, 1075-1082. [CrossRef] [PubMed]

42. Freund, E.; Liedtke, K.R.; van der Linde, J.; Metelmann, H.R.; Heidecke, C.D.; Partecke, L.I.; Bekeschus, S. Physical plasma-treated saline promotes an immunogenic phenotype in CT26 colon cancer cells in vitro and in vivo. Sci. Rep. 2019, 9, 634. [CrossRef] [PubMed]

43. Van Loenhout, J.; Flieswasser, T.; Freire Boullosa, L.; De Waele, J.; Van Audenaerde, J.; Marcq, E.; Jacobs, J.; Lin, A.; Lion, E.; Dewitte, H.; et al. Cold Atmospheric Plasma-Treated PBS Eliminates Immunosuppressive Pancreatic Stellate Cells and Induces Immunogenic Cell Death of Pancreatic Cancer Cells. Cancers (Basel) 2019, 11, 1597. [CrossRef] [PubMed]

44. Bernhardt, T.; Semmler, M.L.; Schafer, M.; Bekeschus, S.; Emmert, S.; Boeckmann, L. Plasma Medicine: Applications of Cold Atmospheric Pressure Plasma in Dermatology. Oxid. Med. Cell. Longev. 2019, 2019, 3873928. [CrossRef] [PubMed] 
45. Privat-Maldonado, A.; Schmidt, S.; Lin, A.; Weltmann, K.D.; Wende, K.; Bogaerts, A.; Bekeschus, S. ROS from physical plasmas: Redox chemistry for biomedical therapy. Oxid. Med. Cell. Longev. 2019, 2019. [CrossRef] [PubMed]

46. Mitra, S.; Nguyen, L.N.; Akter, M.; Park, G.; Choi, E.H.; Kaushik, N.K. Impact of ROS Generated by Chemical, Physical, and Plasma Techniques on Cancer Attenuation. Cancers (Basel) 2019, 11, 1030. [CrossRef] [PubMed]

47. Kim, S.J.; Chung, T.H. Cold atmospheric plasma jet-generated RONS and their selective effects on normal and carcinoma cells. Sci. Rep. 2016, 6, 20332. [CrossRef]

48. Jablonowski, H.; von Woedtke, T. Research on plasma medicine-relevant plasma-liquid interaction: What happened in the past five years? Clin. Plasma Med. 2015, 3, 42-52. [CrossRef]

49. Winter, J.; Tresp, H.; Hammer, M.U.; Iseni, S.; Kupsch, S.; Schmidt-Bleker, A.; Wende, K.; Dünnbier, M.; Masur, K.; Weltmann, K.D.; et al. Tracking plasma generated $\mathrm{H} 2 \mathrm{O} 2$ from gas into liquid phase and revealing its dominant impact on human skin cells. J. Phys. D 2014, 47, 285401. [CrossRef]

50. Nakamura, K.; Kajiyama, H.; Peng, Y.; Utsumi, F.; Yoshikawa, N.; Tanaka, H.; Mizuno, M.; Toyokuni, S.; Hori, M.; Kikkawa, F. Intraperitoneal Treatment With Plasma-Activated Liquid Inhibits Peritoneal Metastasis In Ovarian Cancer Mouse Model. Clin. Plas. Med. 2018, 9, 47-48. [CrossRef]

51. Sato, Y.; Yamada, S.; Takeda, S.; Hattori, N.; Nakamura, K.; Tanaka, H.; Mizuno, M.; Hori, M.; Kodera, Y. Effect of Plasma-Activated Lactated Ringer's Solution on Pancreatic Cancer Cells In Vitro and In Vivo. Ann. Surg. Oncol. 2018, 25, 299-307. [CrossRef]

52. Masur, K.; von Behr, M.; Bekeschus, S.; Weltmann, K.D.; Hackbarth, C.; Heidecke, C.D.; von Bernstorff, W.; von Woedtke, T.; Partecke, L.I. Synergistic Inhibition of Tumor Cell Proliferation by Cold Plasma and Gemcitabine. Plasma Process. Polym. 2015, 12, 1377-1382. [CrossRef]

53. Chang, Z.; Li, G.; Liu, J.; Xu, D.; Shi, X.; Zhang, G. Inhibitory effect of non-thermal plasma synergistic Tegafur on pancreatic tumor cell line BxPc-3 proliferation. Plasma Process Polym. 2019, 16, 1800165. [CrossRef]

54. Yan, D.; Xiao, H.; Zhu, W.; Nourmohammadi, N.; Zhang, L.G.; Bian, K.; Keidar, M. The role of aquaporins in the anti-glioblastoma capacity of the cold plasma-stimulated medium. J. Phys. D Appl. Phys. 2017, 50, 055401. [CrossRef]

55. García-Manteiga, J.; Molina-Arcas, M.; Casado, F.J.; Mazo, A.; Pastor-Anglada, M. Nucleoside Transporter Profiles in Human Pancreatic Cancer Cells: Role of hCNT1 in $2^{\prime}, 2^{\prime}$-Difluorodeoxycytidine-Induced Cytotoxicity. Clin. Cancer Res. 2003, 9, 5000-5008. [PubMed]

56. Hagmann, W.; Jesnowski, R.; Lohr, J.M. Interdependence of gemcitabine treatment, transporter expression, and resistance in human pancreatic carcinoma cells. Neoplasia 2010, 12, 740-747. [CrossRef]

57. Ishida, S.; McCormick, F.; Smith-McCune, K.; Hanahan, D. Enhancing tumor-specific uptake of the anticancer drug cisplatin with a copper chelator. Cancer Cell 2010, 17, 574-583. [CrossRef]

58. Bartkova, J.; Rezaei, N.; Liontos, M.; Karakaidos, P.; Kletsas, D.; Issaeva, N.; Vassiliou, L.V.; Kolettas, E.; Niforou, K.; Zoumpourlis, V.C.; et al. Oncogene-induced senescence is part of the tumorigenesis barrier imposed by DNA damage checkpoints. Nature 2006, 444, 633-637. [CrossRef]

59. Cadenas, E.; Davies, K.J. Mitochondrial free radical generation, oxidative stress, and aging. Free Radic. Biol. Med. 2000, 29, 222-230. [CrossRef]

60. Campisi, J. Cancer, aging and cellular senescence. In Vivo 2000, 14, 183-188.

61. Alexander, H.R., Jr.; Li, C.Y.; Kennedy, T.J. Current Management and Future Opportunities for Peritoneal Metastases: Peritoneal Mesothelioma. Ann. Surg. Oncol. 2018, 25, 2159-2164. [CrossRef] [PubMed]

62. Anderson, C.E.; Cha, N.R.; Lindsay, A.D.; Clark, D.S.; Graves, D.B. The Role of Interfacial Reactions in Determining Plasma-Liquid Chemistry. Plasma Chem. Plasma Process. 2016, 36, 1393-1415. [CrossRef]

63. Matsuzaki, T.; Kano, A.; Kamiya, T.; Hara, H.; Adachi, T. Enhanced ability of plasma-activated lactated Ringer's solution to induce A549cell injury. Arch. Biochem. Biophys. 2018, 656, 19-30. [CrossRef] [PubMed]

64. Tanaka, H.; Mizuno, M.; Ishikawa, K.; Toyokuni, S.; Kajiyama, H.; Kikkawa, F.; Hori, M. New Hopes for Plasma-Based Cancer Treatment. Plasma 2018, 1, 150-155. [CrossRef]

65. Bekeschus, S.; Schmidt, A.; Weltmann, K.-D.; von Woedtke, T. The plasma jet kINPen-A powerful tool for wound healing. Clin. Plas. Med. 2016, 4, 19-28. [CrossRef]

66. Liedtke, K.R.; Diedrich, S.; Pati, O.; Freund, E.; Flieger, R.; Heidecke, C.D.; Partecke, L.I.; Bekeschus, S. Cold Physical Plasma Selectively Elicits Apoptosis in Murine Pancreatic Cancer Cells In Vitro and In Ovo. Anticancer Res. 2018, 38, 5655-5663. [CrossRef] 
67. Bekeschus, S.; Freund, E.; Spadola, C.; Privat-Maldonado, A.; Hackbarth, C.; Bogaerts, A.; Schmidt, A.; Wende, K.; Weltmann, K.D.; von Woedtke, T.; et al. Risk Assessment of kINPen Plasma Treatment of Four Human Pancreatic Cancer Cell Lines with Respect to Metastasis. Cancers (Basel) 2019, 11, 1237. [CrossRef] 